\title{
Collinear Mirage Effect Measurement of the Thermal Diffusivity in Ferrofluids
}

\author{
A.L.L. Dantas ${ }^{(a)}$, D.Walton ${ }^{(b)}$ and S.M. Shibli ${ }^{(a) *}$ \\ a)Instituto de Física, Universidade de São Paulo, \\ Caixa Postal 66318, 05315-970, São Paulo, SP, BRAZIL \\ b) Physics Dept., McMaster University \\ Hamilton, Ontario, CANADA L8S 4MI \\ Received 29 October, 1998
}

\begin{abstract}
Thermal diffusivity results obtained for different concentrations of the same ferrofluid are reported. The collinear mirage technique was used for its adequacy in obtaining the thermal properties of semitransparent materials with low thermal diffusivities. The results show that, as the ferrofluid concentration increases, the thermal diffusivity also increases, leading to a different trend compared to those reported for ferronematics. The different behaviors observed can be related to the different geometries of thermal waves propagating through the material.

PACS numbers: 77.84.Nh, 78.20.Nv
\end{abstract}

\section{Introduction}

A ferrofluid is a colloidal suspension of small ferromagnetic particles dispersed in a carrier solvent. It has the fluidity of a homogeneous isotropic liquid and high magnetic susceptibility. These two characteristics make the ferrofluid a material of numerous interesting technical applications, such as technological, medical and academic purposes. [1] - [3] Thus, the interest in characterizing this material has been intense. Optical [4], magnetic [5] and structural [6] analysis, among others, have been focused in an effort of characterizing the ferrofluid properties. Nevertheless, to our knowledge, no attempt to study the thermal diffusivity of ferrofluids has been carried out so far.

For obtaining the thermal diffusivity of this material we have applied photothermal wave physics. Depending of the thermo-optical properties and of the structure of the analysed material, a different technique and theory is indicated. In our case the collinear mirage method is the most adequated one.

The use of the collinear configuration is a reliable photothermal alternative for measuring thermal diffu- sivities of semitransparent materials. [7] This technique was also chosen due to its well known potential of avoiding large errors from experimental parameters, and to its high sensitivity to the thermal properties of the sample. Another important advantage, is due to the existence of simple linear relations between two measurable parameters, whose slope can be used to obtain the thermal diffusivity directly.

The collinear mirage effect is accomplished by the absorption of a modulated exciting beam which heats the medium, generating a refractive index gradient within the sample. A second (probe) laser beam runs, through the sample, collinearly and in a direction opposite to that of the pump beam. The probe beam is deflected by the gradient in the index of refraction due to the thermal gradients generated by the exciting beam. The magnitude of the beam deflection, known as mirage deflection, carries information about the thermal properties of the sample.

In the work reported here we have studied the performance of the collinear mirage technique in measuring the thermal diffusivity of ferrofluids (FF's). We have analysed the linearity obtained from measuring

*(a) Author to whom all correspondence should be addressed 
the phase of the collinear mirage deflection as a function of the spacing between the exciting and probe beams, for a fixed frequency. This model is known as the phase method, [8]-[10] and is indicated for analysing data obtained in collinear mirage geometries.

In Section II this model is presented highlighting its validity and limitations for the calculation of the collinear deflection, which is measured through the set up shown in Sec.III. The linear relation used to retrieve the thermal diffusivity of ferrofluids is pointed out and discussed for the analysis of the experimental results, in Sec.IV. Finally, in Sec.V we present the conclusion of this work comparing it to our reported results of the thermal diffusivities for an anisotropic complex fluid, known as ferronematics, which are lyotropic nematic liquid crystals doped with small amounts of ferrofluids. This material was measured at the same photothermal set up presented here and has been also analysed through a linear relation methodology. The different results observed for these two materials can be related to the way of propagation of thermal waves through the media.

\section{Theory}

A theoretical 3D model for the calculation of the collinear mirage deflection is presented in details elsewhere.[8] From this general theoretical calculation, the authors have found a numerical integration that gives a collinear mirage deflection for the probe beam. There are no known analytical solutions for this measurable magnitude, so this integral has been numerically calculated. [9] This procedure leads to several different linear relations, whose slope give directly the thermal diffusivity, being each one indicated for a specific type of material and experimental set up. To choose the most reliable linear relation, it is necessary to verify the ideal conditions, theoretically and experimentally, for the verification of a specific sample and, after that, establish the limits where this linear relation is affected by the experimental parameters, as pump and probe beam sizes.

We have reported that for anisotropic complex fluids, as ferronematics, these linear relations also apply, but not in a direct way. [11] Here, for this point of view, we have a simpler case, since it is known that ferrofluids is an isotropic material, in the absence of a magnetic field, which is the condition kept throughout this work. So, firstly we know that the material analyzed here is homogeneous and isotropic. Secondly, we assume that the geometrical configuration used extends to an infinite medium.

Fig. 1(a) shows the geometry and parameters involved in the collinear mirage detection. Both the probe and exciting beams are assumed to be single rays directed collinearly to the sample, but in an opposite direction. They are separated by a distance $r_{0}$ and oriented relative to the $\mathrm{X}$ axis by an angle $\theta_{0}$. The radius of the exciting beam was considered to be zero. [9]

The expression for the collinear mirage deflection is given by

$$
\phi_{\mathbf{c}}\left(r_{0}\right)=-\frac{1}{n} \frac{d n}{d T} \int_{0}^{\infty}\left(\frac{\partial T}{\partial r}\right)_{r_{0}} d z \hat{\theta}_{0}
$$

where $n$ is the refractive index and $T$ is the absolute temperature.

For the collinear configuration, in the limit of very low absorption we have used a straightforward way of extracting the thermal diffusivity, $\alpha$, from the data:

The temperature in an infinite medium at a distance $r$ from a cylindrical source of radius $r^{\prime}$ is given in Carslaw and Jaeger. [12]

$$
T\left(r, r^{\prime}, t\right)=\int_{0}^{t} F\left(t^{\prime}\right) \frac{\exp \left(-\frac{r^{2}+r^{\prime 2}}{4 \alpha\left(t-t^{\prime}\right)}\right)}{4 \alpha\left(t-t^{\prime}\right)} I_{0}\left[\frac{r r^{\prime}}{2 \alpha\left(t-t^{\prime}\right)}\right] d t^{\prime}
$$

where $I_{0}$ is the modified Bessel function of the first kind of zero order, and $F\left(t^{\prime}\right)$ is the time dependence of the temperature of the cylindrical source. In our case, the source is a focused laser beam with a gaussian profile of 
radius a. Thus integrating over the beam we obtain

$$
T(r, t)=\int_{0}^{r} e^{-\left(\frac{r^{\prime}}{a}\right)^{2}} d r^{\prime} \int_{0}^{t} F\left(t^{\prime}\right) \frac{\exp \left(-\frac{r^{2}+r^{2}}{4 \alpha\left(t-t^{\prime}\right)}\right)}{4 \alpha\left(t-t^{\prime}\right)} I_{0}\left[\frac{r r^{\prime}}{2 \alpha\left(t-t^{\prime}\right)}\right] d t^{\prime}
$$

If $F\left(t^{\prime}\right)=A e^{i \omega t^{\prime}}$, where $A$ is a constant that depends on the power of the exciting laser and the optical absorption of the medium, and $\omega$ is the modulation angular frequency, it is easy to show that

$$
T(r, t)=A e^{i \omega t} \int_{0}^{r} e^{-\left(\frac{r^{\prime}}{a}\right)^{2}} d r^{\prime} I_{0}\left(\sqrt{i} r^{\prime} \sqrt{\frac{\omega}{2 \alpha}}\right) K_{0}\left(\sqrt{i} r \sqrt{\frac{\omega}{2 \alpha}}\right)
$$

where $K_{0}$ is the modified Bessel function of the second kind of zero order.

a)

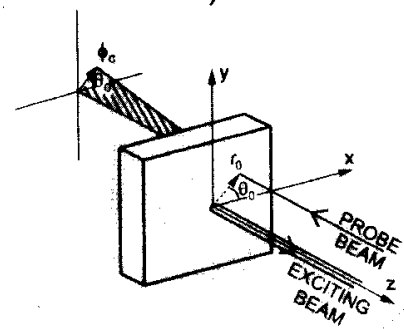

b)

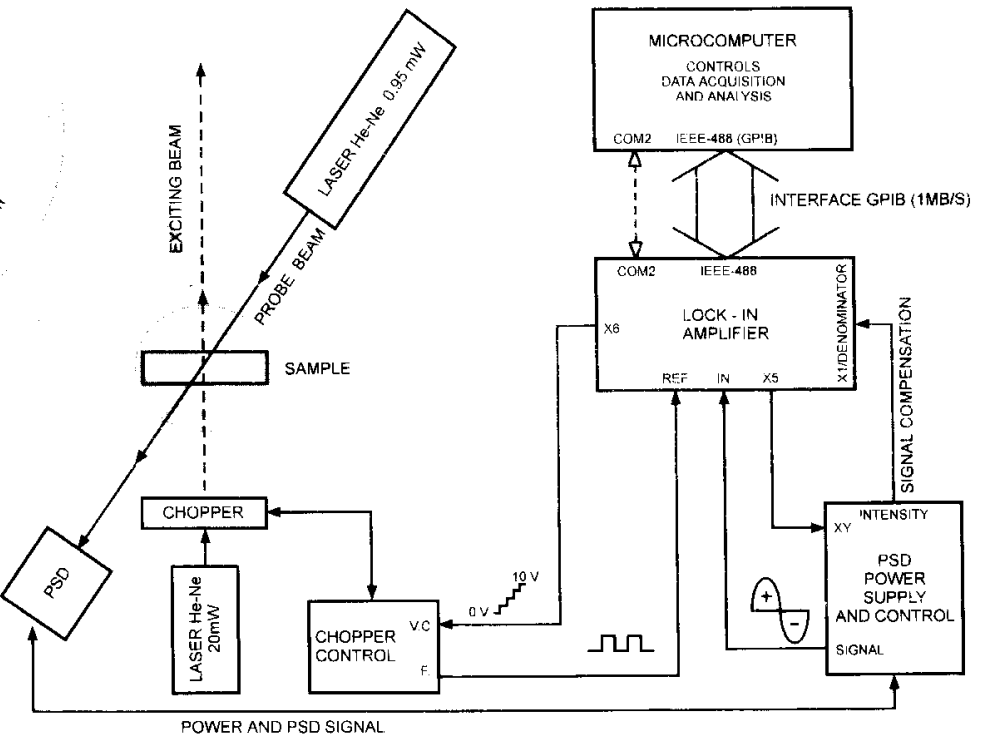

Figure 1. a) Representation of the geometry for the collinear mirage deflection $\phi_{c}$. The probe beam is separated by a distance $r_{0}$ and oriented by an angle $\theta_{0}$ from the exciting beam which is located at coordinates $(0,0)$; b) Schema of the experimental setup used in our collinear mirage technique.

Thus for a given experimental configuration, in which a and $\mathrm{r}$ are kept constant,

$$
T(r, t)=\text { Const. }\left[e^{i \omega t} G\left(\sqrt{\frac{\omega}{2 \alpha}}\right)\right]
$$

where $G$ is some function whose explicit form is not required.

Substituting the above sample temperature expression into Eq.(1), and taking into account the mentioned assumptions, it is possible to obtain $\phi_{c}$ numerically. There is a linear relation when plotting the phase of the collinear deflection $\left[\psi\left(\phi_{c}\right)\right]$ as a function of the separation between the pump and probe beams $r_{0}$, for a fixed frequency. [9] The slope $(m)$ of this linear relation is given by

$$
m=\sqrt{\frac{\omega}{2 \alpha}}
$$

which can be used to calculate the thermal diffusivity $\alpha$. This procedure is known as Phase Method. [8,9]

\section{Experimental}

Ferrofluids are composed by magnetic particles, with typical dimensions of about $10 \mathrm{~nm}$, dispersed in water. [3] Each particle is coated with a dispersive agent to prevent their aggregation. The nanoparticles are magnetic monodomains of magnetite $\left(\mathrm{Fe}_{3} \mathrm{O}_{4}\right)$ purchased from Ferrofluidics Co., with quantities of magnetite, in the solution, of $5.78 \mathrm{~g} / \mathrm{cm}^{3}$. Different concentrations of ferrofluid were used, variyng the volume fractions from $6 \%$ to $10 \%$, in water. The samples were encapsulated in $\mathrm{cu}^{-}$ vettes $0.5 \mathrm{~cm}$ thick on the side and $1.0 \mathrm{~cm}$ large, which allowed us to consider the sample an infinite medium. It is well known [4] that ferrofluids become optically 
anisotropic when subjected to a magnetic field. In this study, the samples were not submitted to a magnetic field, keeping its isotropy. The measurements were performed at room temperature $\left(T=22^{\circ} \mathrm{C}\right)$.

From the thermal measurements in ferrofluids by collinear mirage effect technique we have obtained the mirage signal related to the separation between the pump and probe beams, for a fixed frequency. The deflection of the probe beam, due to the change in the index of refraction, is detected by a position sensitive detector. The curve obtained from plotting the results of the amplitude of the mirage signal as a function of the beams separation was used for calibrating our set up, and has the same shape of the ones reported for measurements in solids. [8, 9]

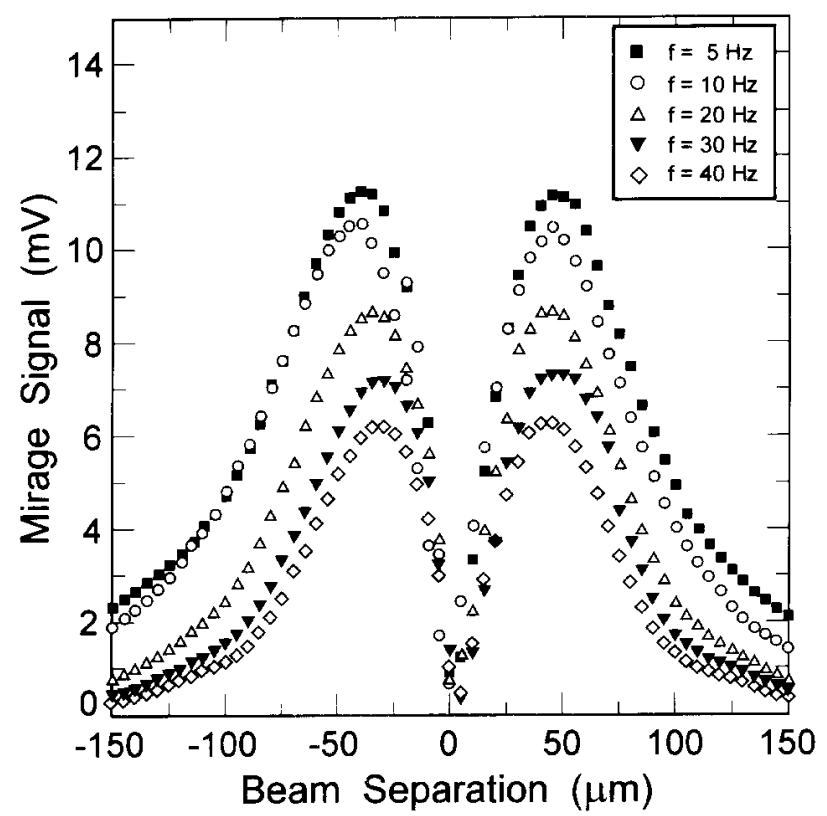

Figure 2. The graph of the mirage signal as a function of the separation between the pump and probe beams, of the variable frequencies ( $f$ ) for the raw data of the ferrofluid sample with the highest ferrofluid concentration. The alignment observed for both peaks at each frequency insures the reliability of the measurements and the calibration of the setup.

A $20 \mathrm{~mW}$ He-Ne laser provided the pump beam, while a $0.95 \mathrm{~mW}$ laser provided the probe beam. Lenses highly focusing were used for both the pump and probe beams, for more accurate measurements. The pump beam was modulated by a mechanical chopper operating between 4 and $400 \mathrm{~Hz}$. The amplitude and phase of the deflected probe beam was provided by a position sensitive detector and a lock-in amplifier connected to the computer through a GPIB interface. This set up, which is shown in Fig.1(b), has been validated by means of experimental measurements on a set of samples with known thermal diffusivities.

To determine the reliability of each measurement, first of all we have measured the mirage signal, which is obtained directly in Volts, from the amplitude values of the lock-in. The signal was then plotted against the beams separation, in micrometers, for each frequency, as seen in Fig. 2.

\section{Results and Discussion}

Plotting the phase of the collinear deflection as a function of the beams separation, for a low frequency, linear relations are attainable. A linear least-squares fit allows us to get the slope $(m)$ of this linear relation and then, by using Eq. (6), the thermal diffusivity. Thus, following always this same procedure, we have been able to retrieve the thermal diffusivity, for a set of samples with different ferrofluid concentration.

Fig. 3 shows the experimental data for one sample with ferrofluid concentration of $10 \%$ by volume, with frequencies values varying from 5 to $40 \mathrm{~Hz}$. This highest doped sample shows the more reliable data throughout the frequency range. Note that the linearity is more evident for the two lowest frequencies. Taking the linear part of these data and applying the phase method, we have obtained directly the thermal diffusivity values for each different sample, in every given frequency.

Comparing the five samples shown in Fig. 4, the thermal diffusivity for a given frequency of $10 \mathrm{~Hz}$, which is the more reliable one with errors of about $5 \%$, we have observed that this thermal parameter decreases with the decrease of the ferrofluid concentration, leading nearly to the water thermal diffusivity. As the ferrofluid concentration increases the thermal diffusivity increases from $(0.223 \pm 0.011) \mathrm{mm}^{2} / \mathrm{s}$ to $(0.268 \pm 0.013) \mathrm{mm}^{2} / \mathrm{s}$. The same trend was observed in an opposite direction in the study of thermal diffusivity in ferronematics, as seen in Table I. Thus, we have seen that the addition of this magnetic fluid at lyotropic liquid crystal lowers the diffusivity up to a certain ferrofluid concentration, for both heat flow parallel and perpendicular to the director. 


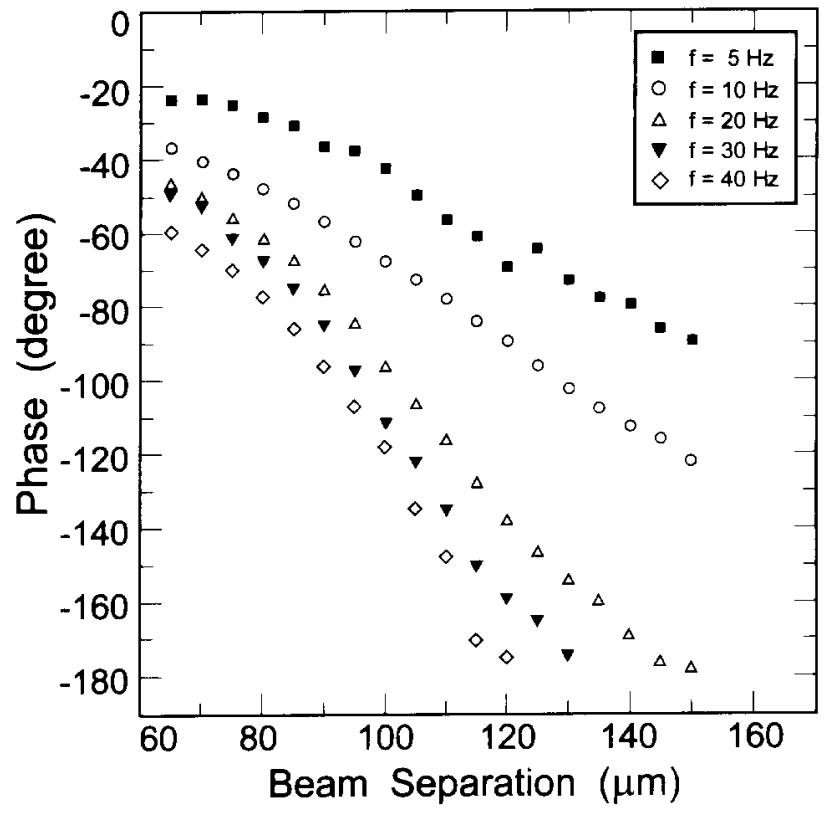

Figure 3. The same data as shown in Fig. 2, for the phase data plotted at the linear part of the beam separation. These data were adjusted until the plotted points coincided with the fitting of a linear slope, used to retrieve the thermal diffusivity. The symbols refer to the same data as that plotted in Fig. 2 .

Then, the influence of the addition of ferrofluid in lyotropic liquid crystals in the nematic phase, to the micellar shape anisotropy and, consequently, to the thermal anisotropy, is related only to the molecular orientation, and not to the ferrofluid addition. Thus, this study shows that the thermal properties of the ferrofluid does not compromise its addition in liquid crystals, during the studies of the thermal properties of ferronematics, as pointed out in our previous work. [11] Though it highligths the importance in the orientation of the micelles, in liquid crystals, for a more accurate thermal result, which seems to affect directly the propagation of the thermal waves through the material. Further analysis of this matter is on its way.

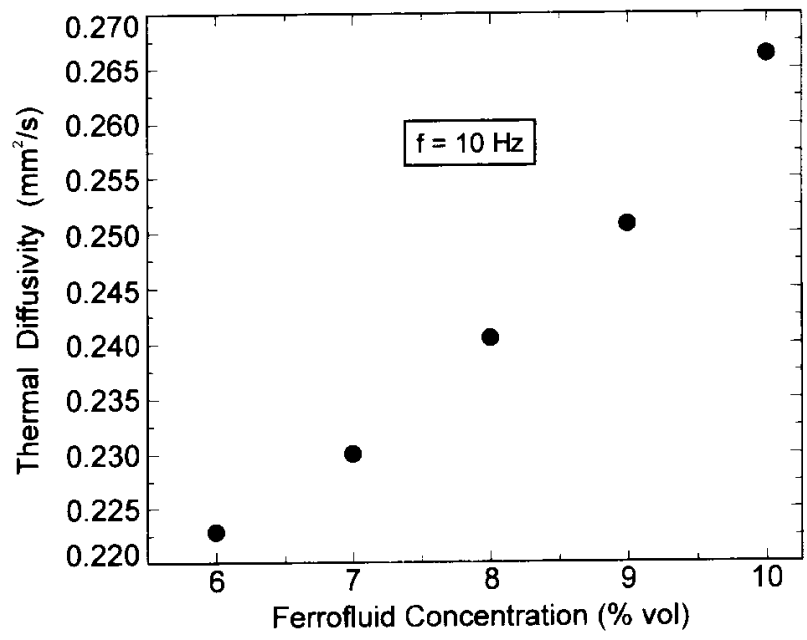

Figure 4. The data of five samples with variable ferrofluid (FF) concentrations, calculated at frequencies of $10 \mathrm{~Hz}$. From this figure we observe the decrease of the thermal diffusivity with the decrease of FF concentration.

Table I. Thermal diffusivities parallel and perpendicular to the director and the ratio between them for the three different ferronematic samples. The ratio shown in the right column is the calculated value for plain lyotropic nematic liquid crystal. [11]

\begin{tabular}{|ccccc|}
\hline $\begin{array}{c}\text { Ferrofluid concentration } \\
\text { (wt \%) }\end{array}$ & $\begin{array}{c}\alpha_{\|} \\
\left(10^{-3} \mathrm{~cm}^{2} / \mathrm{s}\right)\end{array}$ & $\begin{array}{c}\alpha_{\perp} \\
\left(10^{-3} \mathrm{~cm}^{2} / \mathrm{s}\right)\end{array}$ & $\begin{array}{c}\alpha_{\|} / \alpha_{\perp} \\
\text { (measured) }\end{array}$ & $\begin{array}{c}\alpha_{\|} / \alpha_{\perp} \\
\text { (calculated) }\end{array}$ \\
\hline 0.50 & 1.55 & 1.30 & 1.19 & \\
0.75 & 1.40 & 1.15 & 1.22 & 1.27 \\
1.00 & 1.40 & 1.10 & 1.27 & \\
\hline
\end{tabular}

\section{Conclusion}

In conclusion, we have obtained the thermal diffusivities of five samples with different ferrofluid concentration. The results show that for these ferrofluid samples the thermal diffusivity values are higher compared to those already reported for ferronematics. The most interesting result is the opposite trend observed in each case, where we have obtained that the increase of ferrofluid concentration increases the thermal diffusivities in the ferrofluid analysis, but it decreases this same parameter for ferronematic materials. Thus, the ther- mal properties of the ferrofluid do not compromise its addition in liquid crystals, during the studies of the thermal properties of ferronematics. In the other hand, it seems that the orientation of the micelles affects directly the propagation of the thermal waves through the material, which provokes the different behavior in the trend at the thermal diffusivity results as a function of ferrofluid concentration. Finally, we have seen that collinear mirage technique is also a practical and reliable technique for obtaining the thermal diffusivity in transparent isotropic liquid materials as ferrofluids. 


\section{VI acknowledgment}

Financial support received from the Brazilian agency Fundação de Amparo à Pesquisa do Estado de São Paulo - FAPESP is gratefully acknowledged.

\section{References}

[1] R.E. Rosensweig, Ferrohydrodynamics (Cambridge University Press, Cambridge, England, 1985).

[2] Magnetic Fluids and Applications - Handbook, edited by B. Berkovsky and M. Krakov (Begel-House, New York, 1994).

[3] R. Massart, IEEE Trans. Magn. MAG-17, 1247 (1981).

[4] M.F. da Silva and A.M. Figueiredo Neto, Phys. Rev. E 48, 4483 (1993); and references therein.

[5] M.C. Cowley and R.E. Rosensweig, J. Fluid Mech. 30. $671(1967)$.
[6] L.A. do Amaral and F.A.Tourinho, Brazilian J. Phys. 25, 142 (1995); and references therein.

[7] A.C. Boccara, D. Fournier and J. Badoz, Appl. Phys. Lett. 36, 130 (1980).

[8] A. Salazar, A. Sánchez-Lavega and J. Fernández, J. Appl. Phys. 74, 1539 (1993).

[9] A. Salazar and A. Sánchez-Lavega, Rev. Sci. Instrum. 65, 2896 (1994).

[10] A. Salazar, A. Sánchez-Lavega, A. Ocáriz, J. Guitonny, G.C. Pandey, D. Fournier and A.C. Boccara, J. Appl. Phys.79, 3984 (1996).

[11] S.M. Shibli, A.L.L. Dantas and D. Walton, Appl. Phys. Lett. 72, 674 (1998).

[12] H.S. Carslaw and J.C. Jaeger, "Conduction of heat in solids", (Oxford University, London) (1947). 